\title{
Traveling Waves and Nonequilibrium Stationary Patterns in Two-Component Reactive Langmuir Monolayers
}

\author{
Ramon Reigada, ${ }^{1}$ Francesc Sagués, ${ }^{1}$ and Alexander S. Mikhailov ${ }^{2}$ \\ ${ }^{1}$ Departament de Química-Física, Universitat de Barcelona, Avinguda Diagonal 647, 08028 Barcelona, Spain \\ ${ }^{2}$ Abteilung Physikalische Chemie, Fritz-Haber-Institut der Max-Planck-Gesellschaft, Faradayweg 4-6, 14195 Berlin, Germany
}

(Received 4 March 2002; published 28 June 2002)

\begin{abstract}
A simple kinetic model of a two-component phase-separating Langmuir monolayer with a chemical reaction is proposed. Its analysis and numerical simulations show that nonequilibrium periodic stationary structures and patterns of traveling stripes can spontaneously develop. The nonequilibrium phase diagram of this system is constructed and the properties of the patterns are discussed.
\end{abstract}

DOI: 10.1103/PhysRevLett.89.038301

Langmuir monolayers are films formed by organic lipid or amphiphilic molecules disposed on a water-air interface. Such molecular systems provide a classical example of two-dimensional softly condensed matter and are of much interest for biology, because cellular membranes can be viewed as two weakly coupled monolayers. At thermal equilibrium, Langmuir monolayers display a rich variety of structures differing in the spatial order of molecules and in the orientational order of their hydrophobic tails (see [1]). Long-range dipole interactions can furthermore lead to a variety of spatially modulated equilibrium phases [2]. The molecular systems of a living cell are typically found under nonequilibrium conditions, in the presence of chemical reactions and energy flows. Biomembranes are also inhomogeneous and consist of many different species. With this perspective, pattern formation in nonequilibrium multicomponent Langmuir monolayers should be studied. In the experiments by Tabe and Yokoyama [3] (see also [4]), illuminated Langmuir films formed by amphiphilic derivatives of azobencene were investigated using Brewsterangle microscopy. In this system, light of a selected wavelength induced transitions between trans and cis conformations of individual molecules. The physical properties of these two conformations are different, and thus the monolayer essentially represented a nonequilibrium reactive binary mixture. In addition to photoinduced periodic stationary patterns, traveling waves of molecular reorientation resulting from photoisomerization processes were observed. Formation of nonequilibrium stationary Turing-like patterns in reactive phase-separating mixtures is also known for such systems as polymer blends [5-7] and monomolecular adsorbates on metal surfaces $[8,9]$. Periodic patterns of traveling and standing waves, resulting from a Hopf bifurcation with a nonzero wave number, are furthermore possible in three-component reactive phase-separating systems $[10,11]$.

In this Letter, we investigate a model of a twocomponent Langmuir monolayer with orientational order in the presence of a nonequilibrium reversible reaction converting one kind of molecules into another. Our theoretical analysis and numerical simulations reveal
PACS numbers: 82.20.Wt, 47.54.+r, 68.47.Pe, 82.45.Mp

that both Turing-like structures and patterns of traveling waves exist in this greatly simplified model. While not directly explaining the experimental results in Ref. [3], our work provides evidence that traveling wave patterns may represent a general property of Langmuir monolayers subjected to composition changes.

In our model, a Langmuir monolayer is formed by a mixture of two immiscible components $A$ and $B$ with strongly different shapes. Namely, the molecules $B$ are supposed to have a crumpled conformation with only short tails, as compared to molecules $A$. Therefore, molecules $B$ essentially play a role of passive dilution with respect to the orientational ordering of molecules $A$. It is further assumed that a nonequilibrium (e.g., photoinduced) reaction interconverts $A$ and $B$ molecules. Note that $A$ and $B$ may simply represent two isomers of the same molecule. For simplicity, we assume that the forward and reverse reaction rates are equal. Furthermore, we assume that all molecules $A$ have the same and constant azimuth direction, and therefore, only the tilt angle is necessary to describe their orientation. The possibility of spatial two-dimensional (e.g., hexatic) order is also neglected. The total concentration in the monolayer is considered constant, and therefore it is sufficient to specify only the local fraction $c$ of molecules $A$ in the monolayer (the local fraction of molecules $B$ is then given by $1-c$ ).

The evolution equation for $c$ reads

$$
\frac{\partial c}{\partial t}=\frac{D}{k_{B} T} \nabla[c(1-c) \nabla \tilde{\mu}]+K(1-2 c),
$$

where $D$ is the diffusion constant, $T$ is the temperature, $\tilde{\mu}$ is the local chemical potential defined as $\tilde{\mu}=\delta \mathcal{F} / \delta c$, with $\mathcal{F}$ being the free energy functional introduced below. The last term describes the reactions $A \rightarrow B$ and $B \rightarrow A$ with equal rate constants $K$.

Since the molecular subsystem of component $A$ is orientationally ordered, a kinetic equation for the evolution of the order parameter $\eta=\sin \theta$, where $\theta$ is the local average tilt angle of the molecules $A$, should be specified. We assume that this equation is 


$$
\frac{\partial \eta}{\partial t}=-\Gamma \frac{\delta \mathcal{F}}{\delta \eta}-K q(c) \eta
$$

The first term on the right side stands for the relaxation mechanism with a typical relaxation time $\Gamma^{-1}$. The second term takes into account that reactions converting molecules from one conformation to another will also influence the local average tilt. The form of this term and the choice of the function $q(c)$ are discussed later.

The free energy functional of the system reads

$$
\begin{aligned}
\mathcal{F}= & \int\left[-\tilde{\chi}_{0} c^{2}+\frac{1}{2} \tilde{\chi}_{2}(\nabla c)^{2}\right] d x \\
& +k_{B} T \int[\ln c+(1-c) \ln (1-c)] d x \\
& +\int\left[-\frac{1}{2} \tilde{p}(c) \eta^{2}+\frac{1}{4} \tilde{\beta} \eta^{4}+\frac{1}{2} \tilde{g}(\nabla \eta)^{2}\right] d x .
\end{aligned}
$$

The first integral describes lateral interactions between molecules, responsible for phase separation. Note that, for short-range interaction potentials, $\tilde{\chi}_{2}$ can be estimated as $\tilde{\chi}_{2} \approx \frac{1}{2} \tilde{\chi}_{0} r_{0}^{2}$, where $r_{0}$ is the characteristic interaction radius. The second term is the entropy contribution of a two-dimensional lattice gas. The third term is the Landau free energy with respect to the tilt order parameter $\eta$ [1]. Generally, all coefficients in the Landau decomposition should depend on the local concentration $c$. We shall, however, consider only weakly nonuniform states, where local deviations of the concentration $c$ from the uniform stationary state $\bar{c}=1 / 2$ are small. Therefore, we neglect the dependence of the coefficients $\tilde{\beta}$ and $\tilde{g}$ on the variable $c$. On the other hand, the coefficient $\tilde{p}$ of the quadratic term in the Landau free energy is already small near the instability and its dependence on $c$ must be retained, as discussed later.

The equations can be readily adimensionalized: Energy is measured in units of $k_{B} T$, time in units of the relaxational time $\tau_{r} \equiv\left(\Gamma k_{B} T\right)^{-1}$, and space coordinates are rescaled with the relaxational length $L_{r} \equiv \sqrt{D\left(\Gamma k_{B} T\right)^{-1}}$. The model will be then characterized by the dimensionless parameters $\chi_{0}=\tilde{\chi}_{0}\left(k_{B} T\right)^{-1}, \chi_{2}=\tilde{\chi}_{2} \Gamma D^{-1}, \beta=$ $\tilde{\beta}\left(k_{B} T\right)^{-1}, g=\tilde{g} \Gamma D^{-1}$, and $\kappa=K\left(\Gamma k_{B} T\right)^{-1}$. The final equations thus become

$$
\begin{aligned}
& \frac{\partial c}{\partial t}=\nabla^{2} c+\nabla[c(1-c) \nabla \mu]+\kappa(1-2 c), \\
& \frac{\partial \eta}{\partial t}=p(c) \eta-\beta \eta^{3}+g \nabla^{2} \eta-\kappa q(c) \eta,
\end{aligned}
$$

where $p(c)=\tilde{p}(c)\left(k_{B} T\right)^{-1}$ and $\mu$ corresponds to the dimensionless chemical potential,

$$
\mu=-2 \chi_{0} c-\chi_{2} \nabla^{2} c-\frac{1}{2} \frac{d p(c)}{d c} \eta^{2},
$$

once the entropic contribution in $\mathcal{F}$ has been converted into the diffusive term in the first of Eqs. (4). The present model can be viewed as a Cahn-Hilliard equation for the variable $c$ coupled to a relaxational equation for the nonconserved order parameter $\eta$ [12]. The coupling is realized both through the dependence of the control parameter $p$ for the tilt phase transition on concentration $c$ and through a reaction term in the equation for $\eta$.

The stationary uniform states of the system are $\bar{c}=1 / 2$ and $\bar{\eta}= \pm \sqrt{\left(p_{0}-\kappa q_{0}\right) / \beta}$, provided that $p_{0}>\kappa q_{0}$, or $\bar{\eta}=0$, if $p_{0}<\kappa q_{0}$ [here $q_{0}=q(\bar{c})$ and $p_{0}=p(\bar{c})$ ]. The linear stability analysis of the uniform states with respect to small perturbations $\delta c, \delta \eta \propto \exp \left(i k x+\gamma_{k} t\right)$ can be straighforwardly performed. If for the first unstable mode $\omega_{k}=\operatorname{Im}\left(\gamma_{k}\right) \neq 0$, a Hopf bifurcation with a finite wave number is found leading to traveling or standing waves (cf. [13]). On the other hand, if $\omega_{k}=0$ for the first unstable mode, this is a Turing bifurcation leading to stationary periodic patterns.

We first consider instabilities of the uniform tilted phase $(\bar{\eta} \neq 0)$. In this case, the first dimensionless unstable mode for the wave instability is

$$
k_{w}^{2}=\sqrt{\frac{8 \kappa+8 \beta \bar{\eta}^{2}}{\chi_{2}}},
$$

with a frequency that reads

$$
\omega_{w}^{2}=-\left(2 \beta \bar{\eta}^{2}+g k_{w}^{2}\right)^{2}-\frac{k_{w}^{2}}{4} p_{0}^{\prime}\left(p_{0}^{\prime}-\kappa q_{0}^{\prime}\right) \bar{\eta}^{2} .
$$

Here we use the notations $p_{0}^{\prime}=d p / d c, q_{0}^{\prime}=d q / d c$, and $p_{0}^{\prime \prime}=d^{2} p / d c^{2}$, all taken at $c=\bar{c}$. The boundary of the wave instability in the parameter space is given by

$$
\left(\chi_{0}-2+\frac{1}{4} p_{0}^{\prime \prime} \bar{\eta}^{2}-2 g\right)^{2}=8\left(\kappa+\beta \bar{\eta}^{2}\right) \chi_{2},
$$

provided that $\omega_{w}^{2}>0$. Note also that no pure Hopf bifurcation is possible since $\omega_{k}^{2}<0$ for $k_{w}^{2}=0$.

The analysis of the Turing instability of the tilted uniform state yields a cubic equation for $k_{t}^{2}$ which can, however, be reduced to a quadratic equation if $g k^{2} \ll \beta \bar{\eta}^{2}$. When the coefficient $g$ is small (see below), this condition holds, and we obtain that the first unstable mode follows

$$
k_{t}^{2}=\sqrt{\frac{8 \kappa}{\chi_{2}}} .
$$

The boundary of Turing bifurcation is given then by

$$
\left[\chi_{0}-2+\frac{1}{4} p_{0}^{\prime \prime} \bar{\eta}^{2}+\frac{p_{0}^{\prime}}{4 \beta}\left(p_{0}^{\prime}-\kappa q_{0}^{\prime}\right)\right]^{2}=8 \kappa \chi_{2} \text {. }
$$

For the untilted phase $(\bar{\eta}=0)$ only a Turing instability is possible, again with the first unstable mode $k_{t}^{2}=$ $\sqrt{8 \kappa / \chi_{2}}$, and the instability boundary given by

$$
\left(\chi_{0}-2\right)^{2}=8 \kappa \chi_{2} \text {. }
$$

To construct the bifurcation diagram and to perform numerical simulations, functions $p(c)$ and $q(c)$ should be specified. For condensed phases, lowering the lateral pressure of a Langmuir monolayer leads to an increase of its equilibrium tilt [1]. If, as we assume, the molecules $B$ 
play a role of passive dilution for the tilted molecules $A$, increasing $c_{B}$ should be roughly equivalent to decreasing the lateral pressure with respect to the component $A$. Therefore, we choose $p(c)=\pi_{0}+2 \alpha(0.5-c)$, where $\pi_{0}$ determines the equilibrium tilt in the monolayer with $c=\bar{c}=0.5$ and $\alpha$ is a positive coefficient.

The function $q(c)$ should be determined from the kinetic analysis with respect to the orientations of the involved molecules. As an example, we assume that the reaction $B \rightarrow A$, transforming crumpled molecules into the elongated form, is strongly energetically activated (for instance, by light), and therefore new molecules $A$ are created with a random tilt, independently of the tilt of the surrounding molecules. Therefore, the average tilt of newly created molecules $A$ is assumed zero. Introducing the local tilt density $c \eta$, we have that in this case it should obey $\partial_{t}(c \eta)=-K \eta c$. According to it, a loss of $c \eta$ takes place when molecules $A$ transform into $B$. Splitting $\partial_{t}(c \eta)$ and substituting the local variation of $c$ due to reaction, $\partial_{t} c=K(1-2 c)$, leads to $\partial_{t} \eta=-K \eta(1-c) / c$. Thus, in this limit, $q(c)=(1-c) / c$.

In the considered example, $p_{0}=\pi_{0}, p_{0}^{\prime}=-2 \alpha, p_{0}^{\prime \prime}=$ $0, q_{0}=1$, and $q_{0}^{\prime}=-4$. In this case, Eq. (8) for the wave instability boundary reduces to $8 \pi_{0} \chi_{2}=\left(\chi_{0}-2-\right.$ $2 g)^{2}$, and does not include the reaction rate constant $\kappa$. Additionally, the condition $\omega_{w}^{2}>0$ should hold on this boundary. It can be shown that this requirement is satisfied in an interval of $\kappa$ only if the coefficient $g$ is small enough (for the parameters used below it should be smaller than $0.03)$. The boundary of the Turing instability is determined by Eqs. (10) or (11) which do not include $\pi_{0}$.

Figure 1 presents the computed phase diagram in the parameter plane $\left(\pi_{0}, \kappa\right)$ in the limit $g \rightarrow 0$. A uniform tilted phase is found in region III and a uniform nontilted phase is realized in region IVb. Periodic stationary Turinglike nontilted structures are found in region IVa. In region II, stationary structures with periodic variation of both the local concentration and the tilt are expected. Finally, in region I the uniform state is unstable with respect to the spontaneous formation of waves and, thus, traveling or standing waves are expected. Thus, if we keep constant the parameter $\pi_{0}$, determined by the lateral pressure, and increase the reaction rate constant $\kappa$, periodic stationary tilted structures should be first observed. If the tilt is large enough, they will be further replaced by traveling or standing waves which are observed in an interval of $\kappa$. When $\kappa$ is even further increased, periodic stationary nontilted patterns and eventually the uniform nontilted phase should be found. Note that, when the parameter $\chi_{0}$ is increased, the wave instability boundary in Fig. 1 moves upwards and both Turing instability boundaries move to the right.

Numerical integrations of Eqs. (4) in one and two dimensions have been performed using an explicit Euler scheme. Periodic boundary conditions were chosen to model the behavior in a large system far from the boundaries. As initial conditions, small random perturbations

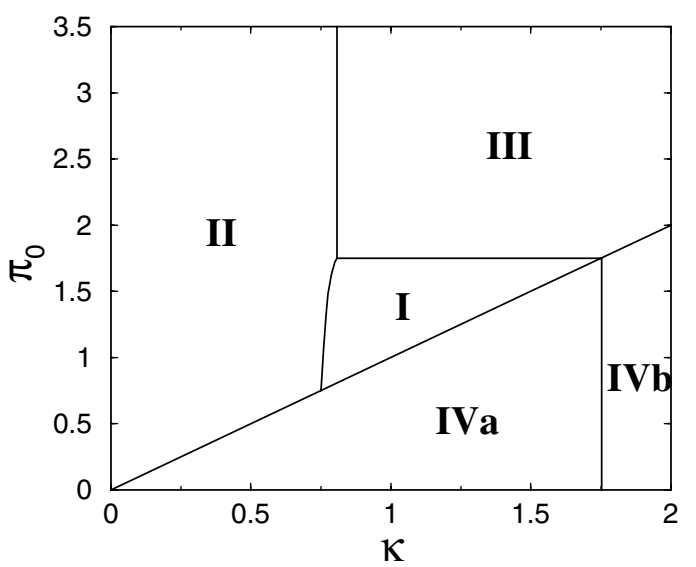

FIG. 1. Phase diagram in the plane $\left(\pi_{0}, \kappa\right)$ for the parameters $\chi_{2}=0.0052, \chi_{0}=2.27, \alpha=1.5$, and $\beta=2$ in the limit $g \rightarrow 0$. The line separating regions I (traveling waves) and II (tilted Turing structures) is determined from numerical one-dimensional simulations; all other boundaries are obtained analytically.

around $\bar{c}$ and $\bar{\eta}$ were taken. The coordinate step $\Delta x$ varied from 0.02 to 0.025 , and the time step was usually $\Delta t=10^{-5}$ to assure a good numerical accuracy.

In Fig. 2 we present the pattern after transients in a typical 1D simulation corresponding to a system in region I. In contrast to a stationary Turing-like structure (not shown here), the distribution of tilt in a traveling wave pattern is shifted with respect to the concentration profile. This asymmetry determines the direction of the wave motion, as indicated in the figure. Note that the spatial period of patterns, $l_{w} \approx 2 \pi / k_{w}=0.82$, is smaller than the diffusion length $L_{\text {diff }}=\sqrt{D / K}$ which is equal to 1 for the chosen set of parameters. In this case, the characteristic interaction radius is $r_{0}=0.067$. Note that, by decreasing the parameter $\chi_{2}$ (and thus decreasing the interaction radius), patterns with characteristic dimensions much smaller than the diffusion length can be obtained.

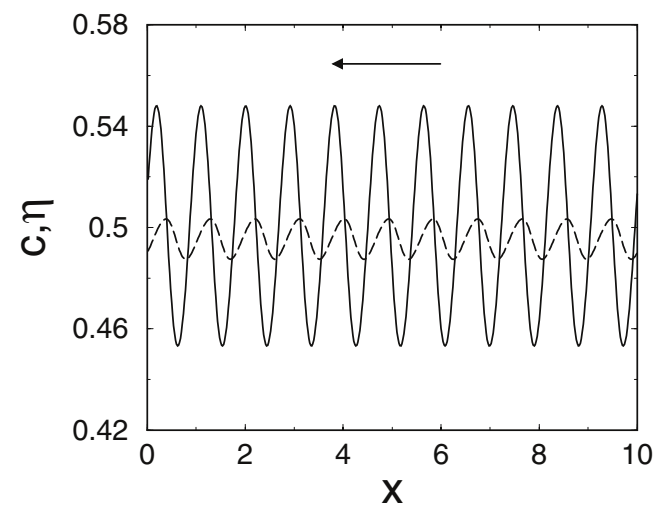

FIG. 2. Spatial distributions of $c$ (solid line) and $\eta$ (dashed line) in a 1D wave pattern. The model parameters are $\kappa=1$ and $\pi_{0}=1.5$. Other parameters are the same as in Fig. 1, except that $g=0.002$. 


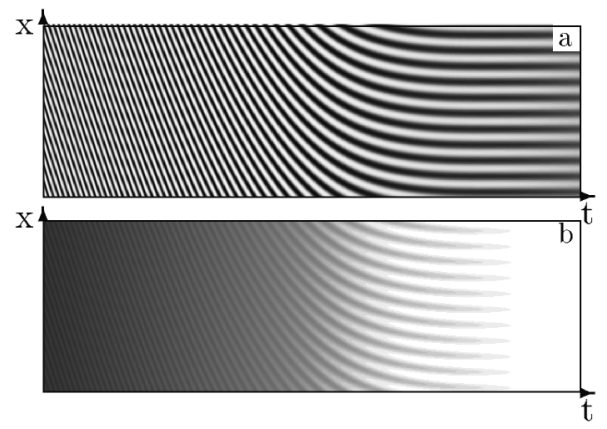

FIG. 3. Temporal evolution of (a) the concentration $c$ and (b) the tilt $\eta$ under a gradual increase of $\kappa$ from 1.24 at $t=30$ to 1.72 at $t=90$. Other parameters and the system size are the same as in Fig. 2. In the used gray-scale representation, darker regions correspond to higher values of the displayed variables.

In the simulation presented in Fig. 3, the coefficient $\kappa$ was slowly increased at a constant speed from 1 to 1.8 within time 100 while keeping all other parameters fixed. This corresponds to traversing the phase diagram (Fig. 1) along a horizontal line at $\pi_{0}=1.5$. We see that, while the wavelength of the traveling waves remains approximately constant, their velocity gradually decreases, and eventually they transform into a stationary Turing-like pattern. This transition is observed near the boundary between the regions I and IVa, with a short delay explained by the nonadiabatic nature of the pattern evolution. The Turing patterns in region IVa should have zero tilt. Indeed, the variable $\eta$ decreases and vanishes (Fig. 3b) as the boundary is crossed.

Figure 4 shows the evolution of a 2D system for the same set of parameters as in Fig. 2. Three snapshots $(4 a-4 c)$ display the concentration distribution at three different times. To visualize the dynamical behavior of the system, the temporal evolution along a one-dimensional cross section is presented below. At the initial stage, a pattern of standing waves develops which later gives rise to a system of domains filled with the waves that travel in different directions. Later on, the system organizes in a coherent wave train traveling at a constant velocity. However, after some time (not shown in the figure), the stripes of the wave train undergo irregular variations and a disordered pattern of traveling waves with defects and dislocations appears. This may imply a transverse modulational instability of traveling stripes, previously discussed in the context of the Bénard convection problem [14]. A detailed investigation of such secondary instabilities of traveling waves will be published separately.

In summary, we have constructed and investigated a phenomenological kinetic model for a Langmuir monolayer consisting of two reactive components that undergo phase separation. Though the model includes strong simplifications, it already predicts nonequilibrium reaction-

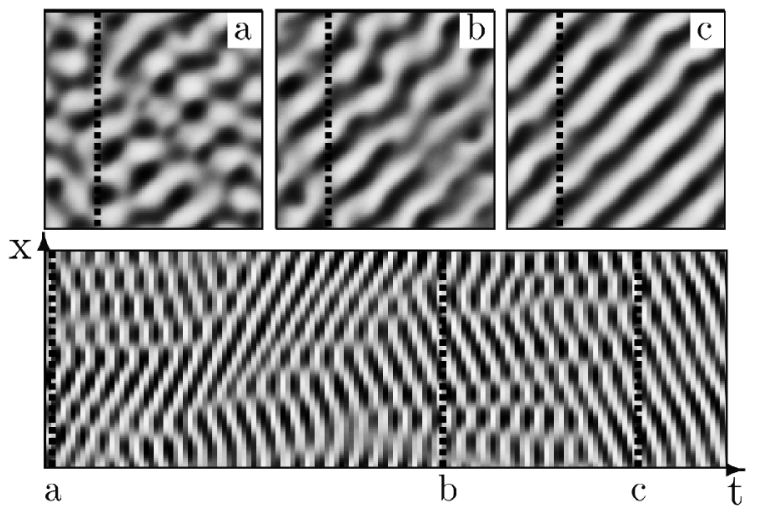

FIG. 4. Temporal evolution of the concentration $c$ in a twodimensional system of linear size $€=10$ and the same parameters as in Fig. 2. The snapshots correspond to times $t=40$ (a), $t=80$ (b), and $t=100$ (c). The bottom panel displays the temporal evolution in the one-dimensional cross section indicated by vertical dashed lines in the snapshots.

induced patterns of stationary or traveling stripes, analogous to those observed in the earlier experiments [3]. This suggests that formation of nonequilibrium traveling structures may be a general property of reactive multicomponent Langmuir monolayers. To obtain detailed understanding of these phenomena, further studies of kinetic reaction processes in such systems are needed.

The authors acknowledge discussions with $\mathrm{M}$. A. Osipov. The support from the DGI (Spain) through Project No. BXX2000-0638 is acknowledged.

[1] V. M. Kaganer, H. Möhwald, and P. Dutta, Rev. Mod. Phys. 71, 779 (1999).

[2] M. Seul and D. Andelman, Science 267, 476 (1995).

[3] Y. Tabe and H. Yokoyama, Langmuir 11, 4609 (1995).

[4] A. S. Mikhailov and G. Ertl, Science 272, 1596 (1996).

[5] S. C. Glotzer, E. A. Di Marzio, and M. Muthukumar, Phys. Rev. Lett. 74, 2034 (1995).

[6] M. Motoyama and T. Ohta, J. Phys. Soc. Jpn. 66, 2715 (1997).

[7] Q. Tran-Cong and A. Harada, Phys. Rev. Lett. 76, 1162 (1996).

[8] J. Verdasca, P. Borckmans, and G. Dewel, Phys. Rev. E 52, R4616 (1995).

[9] M. Hildebrand, A. S. Mikhailov, and G. Ertl, Phys. Rev. E 58, 5483 (1998).

[10] M. Hildebrand, A. S. Mikhailov, and G. Ertl, Phys. Rev. Lett. 81, 2602 (1998).

[11] T. Okuzono and T. Ohta, Phys. Rev. E 64, 045201 (2001).

[12] A. J. Bray, Adv. Phys. 43, 357 (1994).

[13] D. Walgraef, Spatio-Temporal Pattern Formation (Springer-Verlag, New York, 1997).

[14] G. D. Granzow and H. Riecke, Physica (Amsterdam) 249A, 27 (1998). 